\title{
PET neuroimaging reveals histone deacetylase dysregulation in schizophrenia
}

\author{
Tonya M. Gilbert,, Nicole R. Zürcher, ${ }^{1}$ Christine J. Wu, ${ }^{1}$ Anisha Bhanot,, Baileigh G. Hightower, ${ }^{1}$ Minhae Kim, ${ }^{1}$ Daniel S. Albrecht, ${ }^{1}$ \\ Hsiao-Ying Wey, ${ }^{1}$ Frederick A. Schroeder, ${ }^{1}$ Anais Rodriguez-Thompson, ${ }^{1}$ Thomas M. Morin, ${ }^{1}$ Kamber L. Hart, ${ }^{2}$ \\ Amelia M. Pellegrini, ${ }^{2}$ Misha M. Riley, ${ }^{1}$ Changning Wang, ${ }^{1}$ Steven M. Stufflebeam, ${ }^{1}$ Stephen J. Haggarty, ${ }^{2,3,4}$ \\ Daphne J. Holt, ${ }^{1,4}$ Marco L. Loggia, ${ }^{1}$ Roy H. Perlis, ${ }^{2,4,5}$ Hannah E. Brown, ${ }^{4}$ Joshua L. Roffman, ${ }^{1,4}$ and Jacob M. Hooker
}

'Athinoula A. Martinos Center for Biomedical Imaging, Department of Radiology, Massachusetts Ceneral Hospital, Harvard Medical School, Charlestown, Massachusetts, USA. ${ }^{2}$ Center for Cenomic Medicine, ${ }^{3}$ Department of Neurology, and ${ }^{4}$ Department of Psychiatry, Massachusetts General Hospital, Harvard Medical School, Boston, Massachusetts, USA. ${ }^{5}$ Stanley Center for Psychiatric Research, Broad Institute of MIT and Harvard, Cambridge, Massachusetts, USA.

BACKGROUND. Patients with schizophrenia (SCZ) experience chronic cognitive deficits. Histone deacetylases (HDACs) are enzymes that regulate cognitive circuitry; however, the role of HDACs in cognitive disorders, including SCZ, remains unknown in humans. We previously determined that HDAC2 mRNA levels were lower in dorsolateral prefrontal cortex (DLPFC) tissue from donors with SCZ compared with controls. Here we investigated the relationship between in vivo HDAC expression and cognitive impairment in patients with SCZ and matched healthy controls using [ $\left.{ }^{11} \mathrm{C}\right]$ Martinostat positron emission tomography (PET).

METHODS. In a case-control study, relative ["1 C]Martinostat uptake was compared between 14 patients with SCZ or schizoaffective disorder (SCZ/SAD) and 17 controls using hypothesis-driven region-of-interest analysis and unbiased whole brain voxel-wise approaches. Clinical measures, including the MATRICS consensus cognitive battery, were administered.

RESULTS. Relative HDAC expression was lower in the DLPFC of patients with SCZ/SAD compared with controls, and HDAC expression positively correlated with cognitive performance scores across groups. Patients with SCZ/SAD also showed lower relative HDAC expression in the dorsomedial prefrontal cortex and orbitofrontal gyrus, and higher relative HDAC expression in the cerebral white matter, pons, and cerebellum compared with controls.

CONCLUSIONS. These findings provide in vivo evidence of HDAC dysregulation in patients with SCZ and suggest that altered HDAC expression may impact cognitive function in humans.

FUNDING. National Institute of Mental Health (NIMH), Brain and Behavior Foundation, Massachusetts General Hospital (MCH), Athinoula A. Martinos Center for Biomedical Imaging, National Institute of Biomedical Imaging and Bioengineering (NIBIB), NIH Shared Instrumentation Grant Program.

\section{Introduction}

Epigenetic mechanisms have been associated with cognitive function through postmortem human and preclinical studies (1-3), but no direct associations have been made in the living human brain. This is because until recently (4), epigenetic neuroimaging tools did not exist. The epigenetic enzymes that control gene transcription could cause or respond to cell pathology, potentially leading to cognitive deficits in human diseases as diverse as schizophrenia

Conflict of interest: Intellectual property (IP) has been filed around ["C]Martinostat by JMH, CW, and FAS. A portion of this IP has been licensed. SJH has financial interests in Rodin Therapeutics and is an inventor on HDAC inhibitor-related IP licensed to this entity that is unrelated to the present study. JMH is a co-founder of Eikonizo Therapeutics Inc. and consults for Psy Therapeutics Inc. FAS is a co-founder and current employee of Eikonizo Therapeutics Inc. TMG is a current employee of Eikonizo Therapeutics Inc. License: Copyright 2019, American Society for Clinical Investigation.

Submitted: July 23, 2018; Accepted: November 2, 2018.

Reference information: / Clin Invest. 2019;129(1):364-372. https://doi.org/10.1172/JCI123743.
(SCZ) and Alzheimer's disease. Using neuroimaging tools designed to measure the distribution of epigenetic enzymes, we can now begin to explore the relationships among the amount and location of enzymes, brain anatomy and function, and disease phenotypes. Of the epigenetic enzymes that can regulate gene transcription and influence behavior, histone deacetylases (HDACs) have emerged as potential targets for therapeutic interventions (5-8). In the healthy brain normal HDAC activity is critical for maintenance of neural cell identity, survival, and the activity-dependent regulation of neuroplasticity $(2,9-11)$. Using $\left[{ }^{[1} \mathrm{C}\right]$ Martinostat (12-14), an HDAC radiotracer, and positron emission tomography (PET), we previously mapped HDAC expression in healthy individuals (4). Here we measure relative in vivo HDAC expression in the brain of patients with SCZ compared with healthy controls.

Preclinical studies link HDACs to cognitive circuitry. Abnormal amounts of HDACs disrupt cognition in rodents, evidenced through both molecular and behavioral phenotypes. For example, overexpression of HDAC2 in the mouse hippocampus 


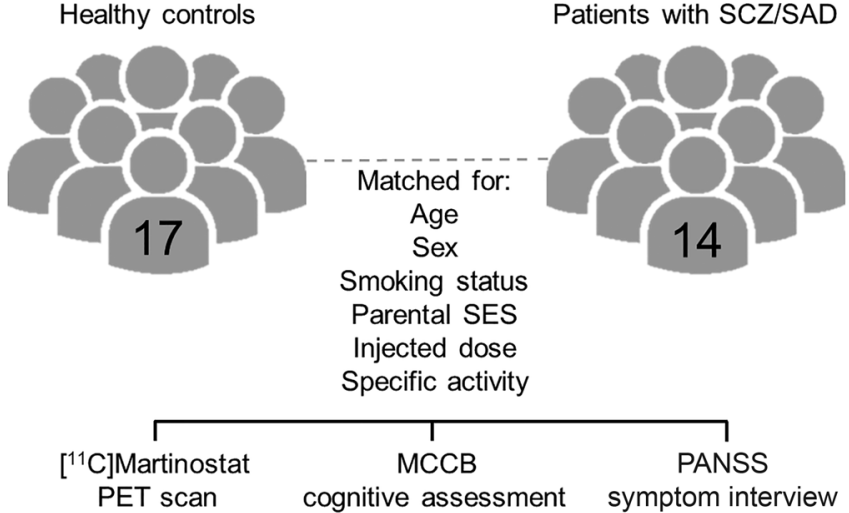

decreased synaptic protein density, impaired long-term potentiation, and negatively regulated memory formation, while $\mathrm{Hdac2}$ deletion had opposing effects (15). Analogously, Hdac2 deletion in postmitotic forebrain neurons enhanced working memory and extinction learning (16). Furthermore, genetic mutation or knockdown of Hdac2 in the central nervous system improved spatial, associative, and threat recognition memory in mouse models of Pitt-Hopkins syndrome $\left(T c f 4^{+/}\right)$(17) and Alzheimer's disease (CK-p25) $(18,19)$. Encouragingly, small molecule HDAC inhibitors conferred cognitive benefits in rodent models of aging $(20,21)$, neurodegeneration $(6,21-26)$, and neuropsychiatric (27-34) disease. Taken together with observations of aberrant HDAC levels in postmortem human brain tissue from donors with Alzheimer's disease $(18,19,35)$, SCZ (29, 36-40), depression $(28,37)$, and bipolar disorder $(39)$, these findings suggest that HDAC-related mechanisms may play a fundamental role in human cognition.

The dorsolateral prefrontal cortex (DLPFC) is a brain region highly important in human cognition that contributes to executive functioning, including working memory, planning, and mental flexibility (41-44). Previously, we observed that HDAC2 mRNA levels were $32 \%$ lower in the DLPFC of donors with SCZ $(n=175)$ compared with controls $(n=210)$, with no difference in HDAC1 mRNA levels (37). The HDAC2 deficit was unique to donors with SCZ among the psychiatric disorders tested, including bipolar disorder $(n=61)$ and major depressive disorder $(n=135)$ (37). Based on these data we hypothesized that regional $\left[{ }^{[1} \mathrm{C}\right]$ Martinostat uptake would be lower in the DLPFC of patients with SCZ. However, other groups observed higher HDAC1 mRNA levels in the prefrontal cortex ( $n=16-27$ per group) $(36,38)$, as well as in GABAergic hippocampal neurons ( $n=7$ per group) (39) from donors with SCZ compared with controls. As studies using postmortem tissue are limited by both physiological differences and spatial sampling constraints, this underscores the potential utility of $\left[{ }^{11} \mathrm{C}\right]$ Martinostat PET for measuring in vivo HDAC expression across the whole brain in patients with SCZ.

Moreover, SCZ represents an example of a chronic cognitive disorder, as more than $75 \%$ of patients show a wide range of cognitive deficits, with mean effect sizes one standard deviation below population means (45). Cognitive impairment manifests early during the course of $\mathrm{SCZ}$ and typically persists, in part because current antipsychotic regimens do not affect cognition $(45,46)$.
Figure 1. Study design. Subjects with SCZ/SAD were group matched to healthy controls based on age, sex, smoking status, and parental socioeconomic status (SES). Injected dose and specific activity of $\left[{ }^{11} \mathrm{C}\right]$ Martinostat did not differ between groups. All subjects underwent a $\left.{ }^{[1} \mathrm{C}\right]$ Martinostat PET scan and cognitive testing with the MCCB. Subjects with SCZ/SAD received the PANSS interview to measure symptom severity.

The severity of cognitive deficits strongly impacts functional outcomes including quality of life $(45,46)$. Thus, amelioration of this highly debilitating form of cognitive impairment represents an important unmet need for SCZ treatment (46).

We find that patients with SCZ/schizoaffective disorder (SAD) show differential $\left[{ }^{11} \mathrm{C}\right]$ Martinostat brain uptake patterns compared with healthy controls, and regional $\left[{ }^{11} \mathrm{C}\right]$ Martinostat brain uptake correlates with cognitive performance scores.

\section{Results}

Study participants. Written informed consent was received from participants prior to inclusion in the study $(n=22$ subjects per group were enrolled). Eligible subjects with SCZ or SAD ( $n=14$, ages 22-65 years) were group matched to eligible healthy control subjects ( $n=17$, ages 23-65 years) on the basis of age, sex, smoking status (47), and parental socioeconomic status (Figure 1 and Table 1). Subjects with SCZ/SAD represented a chronic, medicated cohort with moderate symptom severity as determined by the Positive and Negative Syndrome Scale (PANSS) interview (48) (Table 2). As expected, subjects with SCZ/SAD demonstrated cognitive deficits and scored significantly lower than controls on multiple domains of the NIMH Measurement and Treatment Research to Improve Cognition in Schizophrenia (MATRICS) consensus cognitive battery (MCCB) (49-51) (unpaired $t$ test, $P<0.05$ ) (Table 1 ).

Hypothesis-driven a priori analysis of $\left[{ }^{11} \mathrm{C}\right]$ Martinostat uptake in subjects with SCZ/SAD compared with controls. $\left[{ }^{11} \mathrm{C}\right]$ Martinostat is a radiotracer selective for HDAC paralogs 1, 2, 3, and putatively 6 $(4,12-14)$. $\left[{ }^{11} \mathrm{C}\right]$ Martinostat PET was performed to assess relative in vivo HDAC expression, with standard uptake value (SUV) normalized to whole brain mean (SUVR) used as the primary outcome measure. We previously determined that HDAC2 mRNA levels were significantly lower in postmortem DLPFC tissue from donors with SCZ compared with controls (37). Therefore, we selected the DLPFC for a priori region-of-interest (ROI) analysis in the present imaging study (Figure 2A). In accordance with our previous postmortem data (unpaired $t$ test, $P<0.0001$ ), $\left[{ }^{11} \mathrm{C}\right]$ Martinostat SUVR in the DLPFC was significantly lower in subjects with SCZ/SAD compared with controls (unpaired $t$ test, $P=0.0017$ ) (Figure 2, B and C). Correlations between SUVR in the DLPFC and cognitive performance scores were assessed. SUVR was positively associated with overall composite (Spearman's $r=0.36, P=0.048$ ), speed 


\section{Table 1. Demographic characteristics of study participants}

$\begin{array}{llll}\text { Demographics } & \text { Controls }(\boldsymbol{n}=17) & \operatorname{SCZ/SAD}(\boldsymbol{n}=14) & \boldsymbol{P} \text { value } \\ \text { Age (yr) } & 47.3 \pm 13.1 & 46.3 \pm 13.7 & 0.837 \\ \text { Sex (F:M) } & 8: 9 & 6: 8 & \mathrm{~N} / \mathrm{A} \\ \text { Current tobacco smoker (Y:N) } & 1: 16 & 1: 13 & \mathrm{~N} / \mathrm{A} \\ \text { Parental socioeconomic index } & 3.00 \pm 1.06 & 2.62 \pm 1.45 & 0.428 \\ \text { Injected dose (mCi) } & 5.08 \pm 0.329 & 5.04 \pm 0.278 & 0.706 \\ \text { Molar activity (mCi/nmol) } & 2.10 \pm 0.817 & 2.24 \pm 1.39 & 0.748 \\ \text { MCCB processing T-score } & 52.2 \pm 11.8 & 41.0 \pm 16.9 & 0.0480^{\mathrm{A}} \\ \text { MCCB attention T-score } & 48.8 \pm 11.0 & 43.1 \pm 16.6 & 0.283 \\ \text { MCCB working memory T-score } & 47.9 \pm 11.3 & 37.5 \pm 11.5 & 0.0176^{\mathrm{A}} \\ \text { MCCB verbal learning T-score } & 49.4 \pm 5.34 & 40.1 \pm 15.3 & 0.0448^{\mathrm{A}} \\ \text { MCCB visual learning T-score } & 58.3 \pm 10.3 & 45.5 \pm 16.5 & 0.0197^{\mathrm{A}} \\ \text { MCCB reasoning T-score } & 47.1 \pm 10.6 & 46.2 \pm 11.1 & 0.819 \\ \text { MCCB social cognition T-score } & 45.2 \pm 11.3 & 40.6 \pm 20.3 & 0.452 \\ \text { MCCB overall T-score } & 49.5 \pm 12.0 & 36.9 \pm 19.7 & 0.0493^{\mathrm{A}}\end{array}$

Subjects with SCZ/SAD $(n=14)$ and healthy control subjects $(n=17)$ were group matched based on age, sex, smoking status, and parental socioeconomic status. Injected dose and specific activity of ["C]Martinostat did not differ between groups. The MCCB was administered to all subjects. $P$ values were determined by unpaired $t$ tests. ${ }^{A}$ Significant $P$ values. Non-ratio values represent mean \pm SD .
$P=0.0083$ ), and verbal learning (Spearman's $r=-0.57, P=0.036)$ MATRICS domains (Supplemental Figure 1C). Other MATRICS domains and PANSS scores did not show significant relationships with SUVR in the cerebral white matter or cerebellum cortex (Spearman's correlation analysis, $P>0.05)$ in subjects with SCZ/SAD.

Investigation of cortical thinning and antipsychotic medication usage. Cortical thickness was measured within the DLPFC (Figure 2A, blue) and within frontal cortex regions as detected by voxel-wise analysis (Figure 3A, blue). No significant differences between subjects with SCZ/SAD and controls were found (unpaired $t$ test, $P>$ 0.05) (Supplemental Figure 2A), demonstrating that cortical thinning did not drive $\left[{ }^{11} \mathrm{C}\right]$ Martinostat uptake alterations in these regions. SUVR in the DLPFC (Figure 2A, blue) and SUVR in regions detected by voxel-wise analysis (Figure 3A, blue or red) did not correlate with chlorpromazine (CPZ)

of processing (Spearman's $r=0.38, P=0.036$ ), and social cognition (Spearman's $r=0.53, P=0.0023$ ) MATRICS domains across groups (Figure 2D). Other MATRICS domains and PANSS scores did not show significant relationships with SUVR in the DLPFC (Spearman's correlation analysis, $P>0.05$ ).

Unbiased whole brain voxel-wise analysis of $\left[{ }^{11} \mathrm{C}\right]$ Martinostat uptake in subjects with SCZ/SAD compared with controls. Exploratory voxel-wise analysis was performed to further localize the DLPFC effect, as well as to evaluate relative $\left[{ }^{11} \mathrm{C}\right]$ Martinostat uptake differences between groups across the whole brain (unpaired $t$ test, $Z>2.3, P_{\text {cluster }}<0.05$ ). SUVR was significantly lower in multiple regions of the frontal cortex, including the DLPFC (specifically the rostral middle frontal gyrus and a portion of the superior frontal gyrus), dorsomedial prefrontal cortex, and orbitofrontal gyrus of subjects with SCZ/SAD compared with controls (Figure 3 and Table 3). SUVR was significantly higher in the cerebral white matter, fusiform gyrus, cerebellum, and pons of subjects with SCZ/SAD compared with controls (Figure 3 and Table 3). At an even stricter statistical threshold (unpaired $t$ test, $n=10,000$ permutations, familywise error rate-corrected $\left.P\left[P_{F W E}\right]<0.05\right)$, SUVR was significantly higher in the cerebral white matter and cerebellum of subjects with SCZ/SAD compared with controls (Supplemental Figure 1, A and B; supplemental material available online with this article; https://doi.org/10.1172/JCI123743DS1). To investigate the potential impact of HDAC expression in these regions, preliminary correlations between SUVR in the cerebral white matter or cerebellum cortex (anatomical ROIs) and cognitive performance scores were assessed in subjects with SCZ/SAD. In the cerebral white matter, but not the cerebellum cortex, SUVR was negatively associated with overall composite (Spearman's $r=-0.64, P=0.015$ ), speed of processing (Spearman's $r=-0.86, P=0.0002$ ), attention vigilance (Spearman's $r=-0.75, P=0.0027$ ), working memory (Spearman's $r=-0.69$, equivalent dose (52), a metric of antipsychotic drug exposure, in subjects with SCZ/SAD (Spearman's correlation analysis, $P>$ 0.05) (Supplemental Figure 2B). These data suggest that antipsychotic medication usage did not drive $\left[{ }^{11} \mathrm{C}\right]$ Martinostat uptake alterations in these regions. As antipsychotic drug exposure was shown to increase HDAC2 protein expression in the frontal cortex $(29,53,54)$ of rodents, $\left[{ }^{11} \mathrm{C}\right]$ Martinostat uptake differences may be more pronounced when comparing controls with medication-free subjects with SCZ/SAD.

\section{Discussion}

Dysfunction of the DLPFC has been consistently associated with executive function deficits in SCZ (42-44). We previously discovered that HDAC2 mRNA levels were lower in postmortem DLPFC tissue from donors with SCZ compared with control donors (37). This result was the basis of our hypothesis that regional $\left[{ }^{[11} \mathrm{C}\right]$ Martinostat uptake would be lower in the DLPFC of patients with SCZ. Through a priori region-of-interest analysis, we found that SUVR in the DLPFC was indeed lower in subjects with SCZ/SAD compared with healthy controls. Using unbiased whole brain voxel-wise analysis, we identified additional regions of the frontal cortex that showed lower SUVR in subjects with SCZ/SAD compared with controls, including the dorsomedial prefrontal cortex and the orbitofrontal gyrus, which have been associated with SCZ pathology (55). We further found that the cerebral white matter, fusiform gyrus, pons, and cerebellar regions important for cognitive processes (56-58) showed higher SUVR in subjects with SCZ/ SAD compared with controls. Subregions of the cerebral white matter and cerebellum cortex displayed the strongest statistical differences between groups, suggesting that HDACs may play a particularly important role in these brain regions. Together these results suggest an HDAC imbalance in the brain of patients with SCZ. However, as our study measures changes in $\left[{ }^{11} \mathrm{C}\right]$ Martinostat uptake relative to the whole brain mean (SUVR), and not absolute 
A



B





C
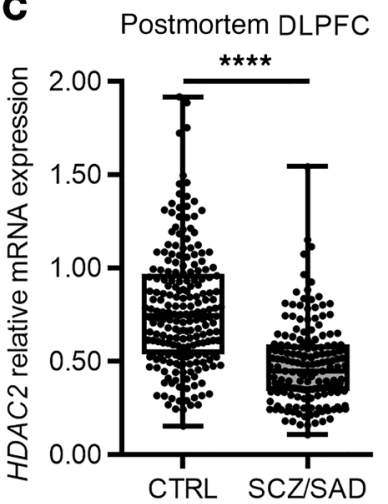

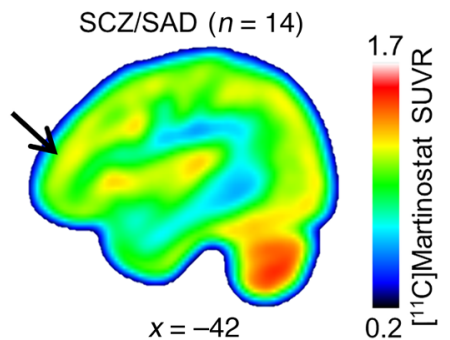

$x=-42$

D



Figure 2. [" $\left.{ }^{11}\right]$ Martinostat SUVR in the DLPFC is lower in subjects with SCZ/SAD compared with healthy matched controls and correlates with cognitive performance scores across groups. (A) ROI mask (left, blue) for the DLPFC and SUVR mean images for $n=17$ controls and $n=14$ subjects with SCZ/SAD at MNI coordinate $x=-42$. (B) Box plot (median, minimum/maximum) depicts SUVR extracted from the DLPFC a priori ROI. ${ }^{* *} P=0.0017$, determined by an unpaired $t$ test. CTRL, control. (C) Data adapted from Schroeder et al. (37). Box plot (median, minimum/ maximum) depicts HDAC2 mRNA expression normalized to a geometric mean ( $\beta$-2-Microglob ulin, $\beta$-Glucuronidase, and $\beta$-Actin) in postmortem DLPFC tissue from $n=210$ control donors and $n=175$ donors with SCZ. ${ }^{* * *} P<0.0001$, determined by an unpaired $t$ test. (D) MCCB T-scores from $n=17$ controls (white circles) and $n=14$ subjects with SCZ/SAD (gray circles) were compared with SUVR extracted from the DLPFC with Spearman's correlation analysis. amounts of $\left[{ }^{11} \mathrm{C}\right]$ Martinostat uptake (distribution volume, $V_{T}$ ), future human postmortem studies and $\left[{ }^{11} \mathrm{C}\right]$ Martinostat PET studies with arterial blood sampling are required to validate HDAC expression differences in these newly identified regions. Our consistent findings in the DLPFC by both imaging and postmortem analyses indicate that these key next steps are warranted.

Subjects with SCZ/SAD exhibited cognitive deficits, including in executive functions, and cognitive performance scores positively correlated with SUVR in the DLPFC across groups. Preliminary findings also showed that in subjects with SCZ/SAD, cognitive performance scores negatively correlated with SUVR in the cerebral white matter. These data may suggest that abnormal HDAC expression, in either direction, could have negative effects on cognition. Alternatively, preclinical evidence suggests that HDAC deficiency may be beneficial in cognitive disorders by restoring neuronal function and promoting synaptic plasticity. A multitude of studies found that HDAC inhibitor treatment enhanced cognitive performance in rodents $(6,15,20-25,27-32)$. Of particular relevance, Covington et al. (28) discovered lower HDAC2 expression in nucleus accumbens tissue from donors with depression compared with control donors, and in a rodent model of depression compared with WT animals. Despite this HDAC2 deficiency, HDAC inhibitor treatment produced antide- pressive effects in the rodent model of depression (28). Future longitudinal PET studies comparing $\left[{ }^{11} \mathrm{C}\right]$ Martinostat uptake with cognitive phenotypes throughout the course of SCZ (59) may help reconcile these hypotheses.

A major challenge presented by imaging and postmortem studies is their mechanistic interpretations. For example, what does a decrease in HDAC in the frontal cortex mean in a brain circuit-based context? To probe the downstream molecular consequences of decreased $\left[{ }^{11} \mathrm{C}\right]$ Martinostat signal, we previously used pharmacologic doses of Martinostat to inhibit HDAC enzyme activity in human neural precursor cells (4). We assayed mRNA levels of genes known to be regulated by HDAC (18) and critical for synaptic plasticity $(60,61)$ such as early growth response 1 (EGR1), brain-derived neurotrophic factor $(B D N F)$, and synaptophysin $(S Y P)$. Notably, EGR1 is also associated with SCZ risk (62), and decreased EGR1 expression has been observed in DLPFC tissue from donors with SCZ $(n=35-62)(62,63)$. Martinostat treatment increased EGR1, BDNF, and SYP gene expression (4). These data could indicate a relationship between HDAC amount and human cognitive circuitry, and may support the potential utility of HDAC inhibitors as therapeutics for SCZ. However, future studies using neurons derived from patients with SCZ and genetic modulation of HDAC expression are required to strengthen this hypothesis (64, 


\section{Table 2. Clinical characteristics of study participants with SCZ and SAD}

\section{Clinical characteristic \\ Psychiatric diagnosis (SCZ:SAD) \\ Second-generation antipsychotics}

First-generation antipsychotics

Antidepressants

Anticonvulsants

CPZ equivalents $(\mathrm{mg} / \mathrm{d})$

PANSS total score

PANSS general symptoms score

PANSS positive symptoms score

PANSS negative symptoms score

The number of subjects taking each medication is indicated in parentheses. CPZ equivalents and PANSS scores represent mean \pm SD.

65). In particular, using induced pluripotent stem cell-derived neuronal models from patients imaged with $\left[{ }^{11} \mathrm{C}\right]$ Martinostat would allow for a unique and powerful interplay between in vivo observations and ex vivo mechanistic insights within the same genetic landscape (66).

In conclusion, our study presents in vivo evidence of human neuroepigenetic dysregulation in SCZ and provides the foundation for using $\left[{ }^{11} \mathrm{C}\right]$ Martinostat PET to study the role of HDACs in human cognition.

\section{Methods}

Study design. Our main research objective was to compare relative $\left[{ }^{11} \mathrm{C}\right]$ Martinostat brain uptake between subjects with SCZ/SAD and groupmatched healthy controls using magnetic resonance-PET (MR-PET). SUV collected 60-90 minutes after radiotracer injection, normalized to whole brain mean (SUVR), was the primary endpoint assessed. Data from 2 subjects were excluded: a subject with SCZ exited the scanner before the 60- to 90-minute time point, and a control subject was found to use psychotropic medications. Imaging studies were not blinded, and no outliers were excluded.

Participants. All participants provided written informed consent. Participants underwent a physical examination with a licensed physician or nurse practitioner. Medical history, smoking status, and medication use were recorded. Illicit drug use was assessed by a urine drug screen (Discover Drug Test Card DIS-DOA-3124, American Screening Corp.). A serum pregnancy test (Sure-Vue serum hCG-STAT, Fisher HealthCare) was performed for female participants of childbearing potential to ensure no pregnancy at the time of the scan.

Inclusion/exclusion criteria for participants. Subjects with a diagnosis of SCZ or SAD were group matched for age, sex, smoking status, and parental socioeconomic status with healthy controls. One subject with SCZ/SAD was unable to provide parental socioeconomic information, and one subject with SCZ/SAD took nicotine replacement therapy and was classified as a non-smoker. At the level of individual pairs, age did not differ by more than 5 years. Diagnosis of SCZ or SAD was confirmed by licensed physician evaluation with the Structured Clinical Interview for DSM-IV-TR Axis I Disorders, Research Version, Patient Edition (SCID-I/P) (67) or psychiatrist consensus. To verify that control subjects did not have a psychiatric disorder, the Structured Clinical Interview for DSM-IV-TR Axis I Disorders, Research Version, Non-Patient Edition (SCID-I/NP) (68) was administered. Subjects were physically healthy as determined by medical history and a physical examination. Subjects had no history of major physical illness (including diabetes mellitus), impaired elimination, major surgery within the past year, or present substance abuse (other than nicotine). Subjects met a multipoint safety checklist for both MR and PET procedures. Subjects who were pregnant or breastfeeding, had ferromagnetic foreign bodies, had a history of major head trauma, or failed the urine drug screen were excluded. Controls with a current/previous psychiatric disorder or history of SCZ, schizophreniform disorder, or schizoaffective disorder in a first-degree relative were excluded. Controls taking psychotropic medications or hormone treatments were excluded.

Clinical assessments and rating scales. Subjects underwent the MATRICS consensus cognitive battery (MCCB) (49-51) to measure cognitive function. Subjects with SCZ/SAD were administered the PANSS interview (48) to measure symptom severity. Our staff was certified by the MGH Schizophrenia Clinical \& Research Program to perform both assessments. The Hollingshead index (69) was used to determine parental socioeconomic status. CPZ equivalents were calculated (52) for subjects with SCZ/SAD. One subject was not regularly taking antipsychotics and was not included in the calculation.

Radiosynthesis of $\left[{ }^{11} \mathrm{C}\right]$ Martinostat. $\left[{ }^{[1} \mathrm{C}\right]$ Martinostat was synthesized as previously described (4).

$M R-P E T$ data acquisition and reconstruction. An intravenous catheter was placed in the antecubital vein of the arm, and $\sim \mathrm{mCi}\left[{ }^{11} \mathrm{C}\right] \mathrm{Mar}-$ tinostat $(5.08 \pm 0.33 \mathrm{mCi}$ for control subjects; $n=17)$ and $(5.04 \pm 0.28$ mCi for subjects with SCZ/SAD; $n=14$ ) was injected as a manual bolus over a period of less than 1 minute by a licensed nuclear medicine technologist. Participants were instructed to remain still for the entire duration of the 90-minute scan. MR and PET images were acquired on a 3T Siemens TIM Trio with a BrainPET insert using an 8-channel head coil (70). An anatomical T1-weighted multi-echo MPRAGE sequence (MEMPRAGE) with echo-planar imaging (EPI) navigator that allows prospective motion correction $(71,72)$ (repetition time [TR], $2530 \mathrm{~ms}$; echo time 1 [TE1], $1.66 \mathrm{~ms}$; TE2, $3.53 \mathrm{~ms}$; TE3, $5.40 \mathrm{~ms}$; TE4, $7.27 \mathrm{~ms}$; inversion time [TI], $1100 \mathrm{~ms}$; flip angle, $7^{\circ}$; and isotropic resolution, $1 \mathrm{~mm}$ ) was acquired. To account for motion between MEMPRAGE acquisition and the PET frame of interest, the MEMPRAGE was realigned to a single reference PET frame 60-90 minutes after radiotracer injection. MR-based attenuation correction was applied using a statistical parametric mapping (SPM), pseudo-computed tomography method (73). PET data were binned into six 5-minute frames spanning 60-90 minutes after radiotracer injection and reconstructed using the three-dimensional ordinary Poisson ordered-subset expectation maximization (3D OP-OSEM) algorithm with detector efficiency, decay, dead time, attenuation, and scatter corrections applied $(74,75)$. The final PET images were reconstructed into 153 slices with $256 \times 256$ pixels and a 1.25-mm isotropic voxel size in the units of SUV. The final MR images were reconstructed with FreeSurfer version 6.0 (http://surfer. nmr.mgh.harvard.edu/) (76). 
A
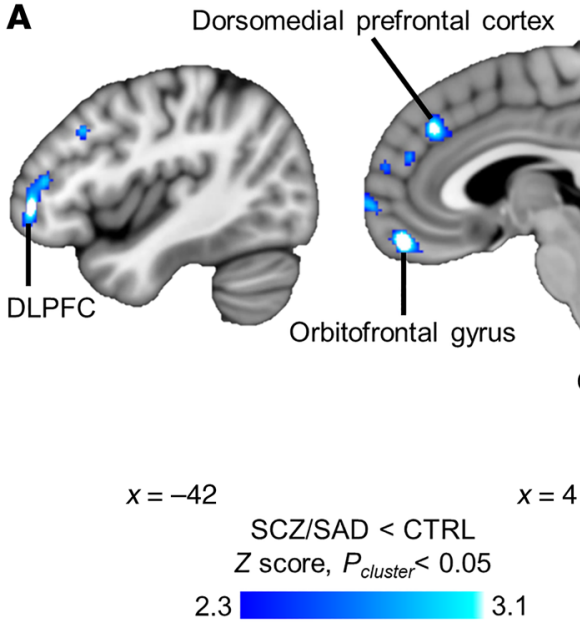

B
Post hoc ROI

from voxel-wise $\downarrow$

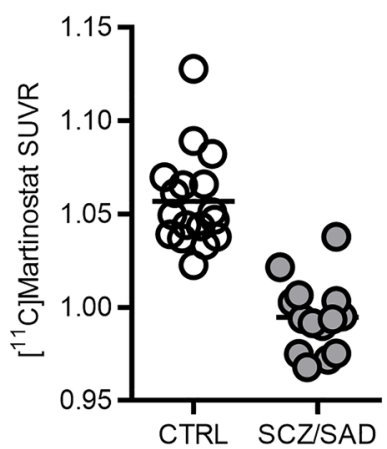

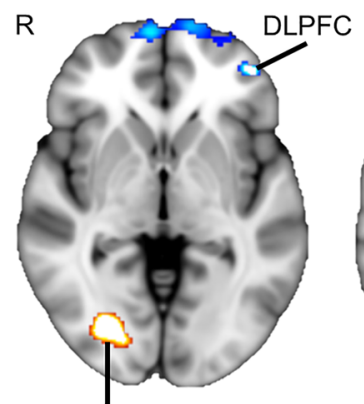

Cerebral white matter /

Fusiform gyrus

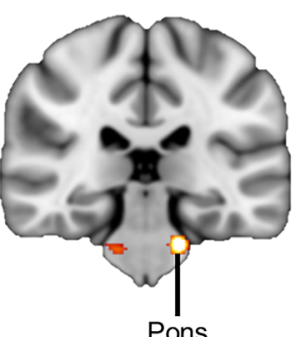

Pons

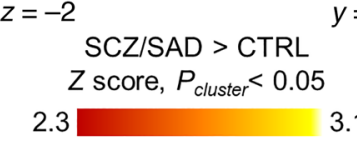

$z=-2$

SCZ/SAD > CTRL

$Z$ score, $P_{\text {cluster }}<0.05$

$2.3 \square 3$.

Post hoc RO

from voxel-wise $\uparrow$

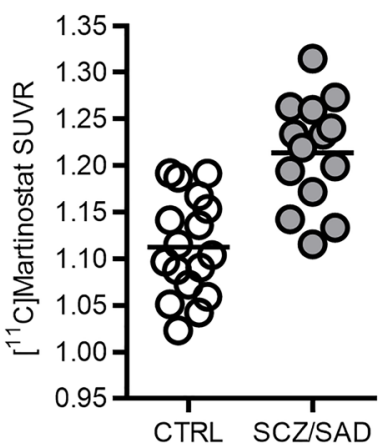

Figure 3. Whole brain voxel-wise analysis identifies additional regions with ["C]Martinostat SUVR differences between subjects with SCZ/SAD and healthy matched controls. (A) $Z$ score statistical maps were created by comparing SUVR between $n=14$ subjects with SCZ/SAD and $n=17$ controls (unpaired $t$ test). $Z$ score maps are overlaid onto the MNI 1-mm template in radiological orientation at MNI coordinates $x=-42, x=4, z=-2$, and $y=-30$. Blue represents regions with significantly lower SUVR, and red represents regions with significantly higher SUVR, in subjects with SCZ/SAD compared with controls ( $Z>2.3$ and $P_{\text {cluster }}<0.05$ ). (B) Scatterplots depicting SUVR extracted from the post hoc lower (blue) and post hoc higher (red) regions are shown for illustrative purposes.

Image analyses. In order to correct for inter-frame motion, the MCFLIRT tool (77-79) in FSL (Oxford Centre for Functional MRI of the Brain [FMRIB] Software Library) version 5.0.7 (77-80) was used. In brief, PET data corresponding to 5-minute bins spanning 60-90 minutes after radiotracer injection were smoothed $6 \mathrm{~mm}$ and aligned to a mean volume image using rigid body linear registration with 6 degrees of freedom. The motion correction matrix was applied to the unsmoothed PET data using FSL's applyxfm4d. PET data were averaged to create a motion-corrected, unsmoothed mean image for each subject, which was then registered to the subject's MEMPRAGE using mri_coreg from FreeSurfer 6.0 (76) and skull-stripped. The MEMPRAGE was registered to Montreal Neurological Institute (MNI) space using linear (FLIRT [FMRIB's linear image registration tool]) $(80,81)$ and nonlinear (FNIRT [FMRIB's nonlinear image registration tool]) (82) algorithms in FSL, and the registration matrix was applied to move the PET image into MNI space. The PET image was intensity normalized to whole brain mean as $\operatorname{SUVR}(83,84)$ to measure relative differences in $\left[{ }^{11} \mathrm{C}\right]$ Martinostat uptake, while controlling for interindividual differences in global signal. The SUVR image was spatially smoothed $8 \mathrm{~mm}$ full width at half maximum. A priori ROI analysis of SUVR was performed between groups for the DLPFC. The DLPFC surface label was based on work by Yendiki et al. (85) and was converted into a volume in MNI space using FreeSurfer's mri label2vol. Whole brain voxel-wise analysis of SUVR was performed between groups using FSL's FEAT with ordinary least squares (OLS) mixed-effects modeling, a significance threshold of $Z>2.3$, and cluster correction of $P_{\text {cluster }}<0.05$ (86). Whole brain voxel-wise analysis was also performed using FSL's randomise with 10,000 permutations, threshold-free cluster enhancement (TFCE), and $P_{F W E}<0.05$. Age and sex were added as nuisance regressors in voxel-wise analyses. Correlations between anatomical ROIs and cognitive performance scores were performed with SUVR images (spatially smoothed $8 \mathrm{~mm}$ full width at half maximum) in native space using masks for the cerebral white matter and cerebellum cortex generated by FreeSurfer's automated parcellation and segmentation (76). FreeSurfer's mri_segstats was used to measure cortical thickness. Anatomical regions were described using Atlas of the Human Brain (87).

Statistics. Demographic variables and MATRICS domains were compared between groups using unpaired 2-tailed $t$ tests (Table 1). For a priori ROI analysis of SUVR or postmortem HDAC2 mRNA comparisons 


\section{Table 3. Whole brain voxel-wise analysis reveals ["1"C]Martinostat SUVR differences between subjects with SCZ/SAD and healthy matched controls}

\begin{tabular}{|c|c|c|}
\hline \multicolumn{3}{|c|}{ Voxel-wise analysis SCZ/SAD < CTRL, cluster size: 3294 voxels, $\boldsymbol{P}_{\text {cluster }}<0.05$} \\
\hline MNI coordinate (mm) & Z score & Anatomical region \\
\hline$x=-16, y=36, z=-24$ & 4.38 & Left medial orbitofrontal gyrus \\
\hline$x=-22, y=32, z=42$ & 4.21 & Left superior frontal sulcus \\
\hline$x=0, y=52, z=-18$ & 4.21 & Inferior rostral gyrus \\
\hline$x=-16, y=68, z=8$ & 4.02 & Left middle frontopolar gyrus \\
\hline$x=-6, y=26, z=32$ & 3.85 & Left superior frontal gyrus, medial part \\
\hline$x=12, y=48, z=-20$ & 3.74 & Right medial orbitofrontal gyrus \\
\hline$x=-42, y=48, z=0$ & 3.64 & $\begin{array}{l}\text { Left inferior frontal gyrus, pars } \\
\text { opercularis }\end{array}$ \\
\hline$x=4, y=36, z=36$ & 3.29 & Right superior frontal gyrus, medial part \\
\hline \multicolumn{3}{|c|}{ Voxel-wise analysis SCZ/SAD > CTRL, cluster size: 4400 voxels, $P_{\text {cluster }}<0.05$} \\
\hline MNI coordinate (mm) & $Z$ score & Anatomical region \\
\hline$x=26, y=-78, z=-2$ & 4.94 & $\begin{array}{l}\text { Right cerebral white matter, occipital } \\
\text { gyrus }\end{array}$ \\
\hline$x=-6, y=-84, z=-28$ & 3.82 & Left cerebellar hemisphere \\
\hline$x=18, y=-56, z=-42$ & 3.71 & Right cerebellar white matter \\
\hline$x=-4, y=-66, z=-24$ & 3.69 & Cerebellar vermis \\
\hline$x=-10, y=-68, z=-36$ & 3.67 & Left cerebellar white matter \\
\hline$x=-16, y=-30, z=-32$ & 3.67 & $\begin{array}{l}\text { Left lateral pons/middle cerebellar } \\
\text { peduncle }\end{array}$ \\
\hline$x=16, y=-76, z=-20$ & 3.23 & Right cerebellar hemisphere \\
\hline$x=14, y=-30, z=-34$ & 2.63 & $\begin{array}{l}\text { Right lateral pons/middle cerebellar } \\
\text { peduncle }\end{array}$ \\
\hline
\end{tabular}

An unpaired $t$ test with cluster thresholding $\left(Z>2.3\right.$ and $\left.P_{\text {cluster }}<0.05\right)$ was performed using SUVR images from $n=14$ subjects with SCZ/ SAD and $n=17$ controls. MNI coordinates, $Z$ statistics, and anatomical regions (87) are listed for areas significantly lower (SCZ/SAD < CTRL; top) or higher (SCZ/SAD > CTRL; bottom) in subjects with SCZ/SAD compared with controls.

between groups in the DLPFC, unpaired 2-tailed $t$ tests were performed (Figure 2, B and C). Spearman's correlation analysis was performed to investigate the association between SUVR in the DLPFC and MATRICS or PANSS scores (Figure 2D). For whole brain voxel-wise SUVR comparisons between groups, an unpaired 2-tailed $t$ test was performed with $Z>2.3$ and $P_{\text {cluster }}<0.05$ correction (Figure 3 and Table 3). For whole brain voxel-wise SUVR comparisons between groups, a nonparametric unpaired 2-tailed $t$ test was also performed with TFCE and $P_{F W E}<0.05$ (Supplemental Figure 1, A and B). Spearman's correlation analysis was performed to investigate the association between SUVR in the cerebral white matter, or SUVR in the cerebellum cortex, and MATRICS or PANSS scores (Supplemental Figure 1C). Cortical thickness was compared between groups using unpaired 2-tailed $t$ tests for the DLPFC and for the post hoc statistically significant area (decreased in SCZ/SAD) revealed by voxel-wise analysis (Supplemental Figure 2A). Spearman's correlation analysis was used to investigate the association between SUVR in the DLPFC, or SUVR in the post hoc statistically significant areas (decreased or increased in SCZ/SAD) revealed by voxel-wise analysis, and CPZ equivalent dose exposure (Supplemental Figure 2B).

Study approval. Ethical permission was obtained from the Partners HealthCare IRB and MGH Radioactive Drug Research Com- mittee. Healthy volunteers were recruited through advertising with flyers, Web announcements, and the Partners Healthcare RSVP for Health and Research Patient Data Registry (RPDR) systems. Healthy volunteers were also recruited from IRB protocols 2011P002311 and 2009P000238. Subjects with SCZ/SAD were recruited through the MGH Freedom Trail Clinic and IRB protocol 2009P000238. All study participants provided written informed consent. Imaging procedures were performed at the Athinoula A. Martinos Center for Biomedical Imaging under IRB protocols 2015P001594 and 2015P002008. No adverse events were reported.

\section{Author contributions}

TMG, FAS, SJH, DJH, JLR, and JMH designed the study. TMG, NRZ, CJW, AB, BGH, MK, HYW, FAS, ART, KLH, AMP, MMR, CW, SMS, HEB, and JLR collected human imaging data and/or accompanying demographic/cognitive testing data. TMG and NRZ analyzed human imaging data, and NRZ, DSA, HYW, TMM, and MLL provided imaging training/tools essential for the study. RHP and HEB provided clinical characterization data. TMG and NRZ performed statistical analyses. TMG and JMH wrote the manuscript. All authors edited the manuscript.

\section{Acknowledgments}

We thank J. Sore and the radiopharmacy team for radiotracer synthesis; G. Arabasz, S. Hsu, and R. Butterfield for assistance with MR-PET imaging; and A. Kendall, M. Wentworth, and N. Nortelus for medical coverage. We are grateful to C. Catana, D. Izquierdo, and D. Chonde for PET image analysis advice; C. McCain, L. Namey, C. Cather, and S. DeCross for clinical assessment training; A. Yendiki for providing the DLPFC ROI label; and M. Strebl for providing image visualization tools. This research received funding from the NIMH (R21 MH11197101A1 to JMH), as well as a Brain and Behavior Foundation Independent Investigator Award (JMH) and Young Investigator Award (TMG). This research also received funding from the MGH Research Scholar's Program (JMH, SJH), MGH Executive Committee on Research (ECOR) Fund for Medical Discovery (TMG), and Athinoula A. Martinos Center for Biomedical Imaging Postdoctoral Pilot Funding (TMG). This research was carried out at the Athinoula A. Martinos Center for Biomedical Imaging, using resources provided by the Center for Functional Neuroimaging Technologies, P41EB015896, a P41 Biotechnology Resource Grant supported by the National Institute of Biomedical Imaging and Bioengineering (NIBIB), NIH. This work also involved the use of instrumentation supported by the NIH Shared Instrumentation Grant Program; specifically, S10RR017208-01A1, S10RR026666, S10RR022976, S10RR019933, S10RR023043, and S10RR023401. The content is solely the responsibility of the authors and does not necessarily represent the official views of Massachusetts General Hospital, Harvard University, and its affiliated academic healthcare centers, or the National Institutes of Health.

Address correspondence to: Jacob M. Hooker, Martinos Center for Biomedical Imaging at Massachusetts General Hospital, 149 13th Street, Suite 2301, Charlestown, Massachusetts 02129, USA. Phone: 617.726.6596; Email: hooker@nmr.mgh.harvard.edu. 
1. Fischer A, Sananbenesi F, Mungenast A, Tsai $\mathrm{LH}$. Targeting the correct HDAC(s) to treat cognitive disorders. Trends Pharmacol Sci. 2010;31(12):605-617.

2. Haggarty SJ, Tsai LH. Probing the role of HDACs and mechanisms of chromatin-mediated neuroplasticity. Neurobiol Learn Mem. 2011;96(1):41-52.

3. Penney J, Tsai LH. Histone deacetylases in memory and cognition. Sci Signal. 2014;7(355):re12.

4. Wey HY, et al. Insights into neuroepigenetics through human histone deacetylase PET imaging. Sci Transl Med. 2016;8(351):351ra106.

5. Egger G, Liang G, Aparicio A, Jones PA. Epigenetics in human disease and prospects for epigenetic therapy. Nature. 2004;429(6990):457-463.

6. Wagner FF, et al. Kinetically selective inhibitors of histone deacetylase 2 (HDAC2) as cognition enhancers. Chem Sci. 2015;6(1):804-815.

7. She $\mathrm{A}$, et al. Selectivity and kinetic requirements of HDAC inhibitors as progranulin enhancers for treating frontotemporal dementia. Cell Chem Biol. 2017;24(7):892-906.e5.

8. Fass DM, et al. Crebinostat: a novel cognitive enhancer that inhibits histone deacetylase activity and modulates chromatin-mediated neuroplasticity. Neuropharmacology. 2013;64:81-96.

9. Liu A, et al. The glial or neuronal fate choice of oligodendrocyte progenitors is modulated by their ability to acquire an epigenetic memory. JNeurosci. 2007;27(27):7339-7343.

10. MacDonald JL, Roskams AJ. Histone deacetylases 1 and 2 are expressed at distinct stages of neuro-glial development. Dev Dyn. 2008;237(8):2256-2267.

11. Montgomery RL, Hsieh J, Barbosa AC, Richardson JA, Olson EN. Histone deacetylases 1 and 2 control the progression of neural precursors to neurons during brain development. Proc Natl Acad Sci U S A. 2009;106(19):7876-7881.

12. Wang $\mathrm{C}$, et al. In vivo imaging of histone deacetylases (HDACs) in the central nervous system and major peripheral organs. JMed Chem. 2014;57(19):7999-8009.

13. Schroeder FA, et al. PET imaging demonstrates histone deacetylase target engagement and clarifies brain penetrance of known and novel small molecule inhibitors in rat. ACS Chem Neurosci. 2014;5(10):1055-1062.

14. Wey HY, Wang C, Schroeder FA, Logan J, Price JC, Hooker JM. Kinetic analysis and quantification of $\left[{ }^{11} \mathrm{C}\right]$ Martinostat for in vivo HDAC imaging of the brain. ACS Chem Neurosci. 2015;6(5):708-715.

15. Guan JS, et al. HDAC2 negatively regulates memory formation and synaptic plasticity. Nature. 2009;459(7243):55-60.

16. Morris MJ, Mahgoub M, Na ES, Pranav H, Monteggia LM. Loss of histone deacetylase 2 improves working memory and accelerates extinction learning. J Neurosci. 2013;33(15):6401-6411.

17. Kennedy AJ, et al. Tcf4 Regulates synaptic plasticity, DNA methylation, and memory function. Cell Rep. 2016;16(10):2666-2685.

18. Gräff J, et al. An epigenetic blockade of cognitive functions in the neurodegenerating brain. Nature. 2012;483(7388):222-226.

19. Yamakawa $\mathrm{H}$, et al. The transcription factor Sp3 cooperates with $\mathrm{HDAC} 2$ to regulate synaptic function and plasticity in neurons. Cell Rep. 2017;20(6):1319-1334.

20. Peleg S, et al. Altered histone acetylation is associated with age-dependent memory impairment in mice. Science. 2010;328(5979):753-756.

21. Benito E, et al. HDAC inhibitor-dependent transcriptome and memory reinstatement in cognitive decline models. JClin Invest. 2015;125(9):3572-3584.

22. Fischer A, Sananbenesi F, Wang X, Dobbin M, Tsai LH. Recovery of learning and memory is associated with chromatin remodelling. Nature. 2007;447(7141):178-182.

23. Kilgore $\mathrm{M}$, et al. Inhibitors of class 1 histone deacetylases reverse contextual memory deficits in a mouse model of Alzheimer's disease. Neuropsychopharmacology. 2010;35(4):870-880.

24. Hockly E, et al. Suberoylanilide hydroxamic acid, a histone deacetylase inhibitor, ameliorates motor deficits in a mouse model of Huntington's disease. Proc Natl Acad Sci U S A. 2003;100(4):2041-2046.

25. Janczura KJ, et al. Inhibition of HDAC3 reverses Alzheimer's disease-related pathologies in vitro and in the 3xTg-AD mouse model. Proc Natl Acad Sci U S A. 2018;115(47):E11148-E11157.

26. Siebzehnrübl FA, et al. Early postnatal behavioral, cellular, and molecular changes in models of Huntington disease are reversible by HDAC inhibition. Proc Natl Acad Sci U S A. 2018;115(37):E8765-E8774.

27. Schroeder FA, et al. A selective HDAC $1 / 2$ inhibitor modulates chromatin and gene expression in brain and alters mouse behavior in two moodrelated tests. PLoS One. 2013;8(8):e71323.

28. Covington HE, et al. Antidepressant actions of histone deacetylase inhibitors. J Neurosci. 2009;29(37):11451-11460.

29. Kurita M, et al. HDAC2 regulates atypical antipsychotic responses through the modulation of mGlu2 promoter activity. Nat Neurosci. 2012;15(9):1245-1254.

30. Schroeder FA, Lin CL, Crusio WE, Akbarian S. Antidepressant-like effects of the histone deacetylase inhibitor, sodium butyrate, in the mouse. Biol Psychiatry. 2007;62(1):55-64.

31. Gräff J, et al. Epigenetic priming of memory updating during reconsolidation to attenuate remote fear memories. Cell. 2014;156(1-2):261-276.

32. Malvaez M, et al. HDAC3-selective inhibitor enhances extinction of cocaine-seeking behavior in a persistent manner. Proc Natl Acad Sci U S A. 2013;110(7):2647-2652.

33. Covington HE, Maze I, Vialou V, Nestler EJ. Antidepressant action of HDAC inhibition in the prefrontal cortex. Neuroscience. 2015;298:329-335.

34. de la Fuente Revenga M, et al. HDAC2-dependent antipsychotic-like effects of chronic treatment with the HDAC inhibitor SAHA in mice. Neuroscience. 2018;388:102-117.

35. Anderson KW, Chen J, Wang M, Mast N, Pikuleva IA, Turko IV. Quantification of histone deacetylase isoforms in human frontal cortex, human retina, and mouse brain. PLoS One. 2015;10(5):e0126592.

36. Sharma RP, Grayson DR, Gavin DP. Histone deactylase 1 expression is increased in the prefrontal cortex of schizophrenia subjects: analysis of the National Brain Databank microarray collection. Schizophr Res. 2008;98(1-3):111-117.

37. Schroeder FA, et al. Expression of HDAC2 but not HDAC1 transcript is reduced in dorsolateral prefrontal cortex of patients with schizophrenia. ACS Chem Neurosci. 2017;8(3):662-668.

38. Bahari-Javan S, et al. HDAC1 links early life stress to schizophrenia-like phenotypes. Proc Natl Acad Sci U S A. 2017;114(23):E4686-E4694.

39. Benes FM, Lim B, Matzilevich D, Walsh JP, Subburaju S, Minns M. Regulation of the GABA cell phenotype in hippocampus of schizophrenics and bipolars. Proc Natl Acad Sci U S A. 2007;104(24):10164-10169.

40. Tang B, Dean B, Thomas EA. Disease- and age-related changes in histone acetylation at gene promoters in psychiatric disorders. Transl Psychiatry. 2011;1:e64.

41. Jansma JM, Ramsey NF, van der Wee NJ, Kahn RS. Working memory capacity in schizophrenia: a parametric fMRI study. Schizophr Res. 2004;68(2-3):159-171.

42. Manoach DS, et al. Schizophrenic subjects activate dorsolateral prefrontal cortex during a working memory task, as measured by fMRI. Biol Psychiatry. 1999;45(9):1128-1137.

43. Manoach DS, et al. Schizophrenic subjects show aberrant fMRI activation of dorsolateral prefrontal cortex and basal ganglia during working memory performance. Biol Psychiatry. 2000;48(2):99-109.

44. Potkin SG, et al. Working memory and DLPFC inefficiency in schizophrenia: the FBIRN study. Schizophr Bull. 2009;35(1):19-31.

45. Cha DS, Kudlow PA, Baskaran A, Mansur $\mathrm{RB}$, McIntyre RS. Implications of epigenetic modulation for novel treatment approaches in patients with schizophrenia. Neuropharmacology. 2014;77:481-486.

46. Forray C, Buller R. Challenges and opportunities for the development of new antipsychotic drugs. Biochem Pharmacol. 2017;143:10-24.

47. Schneider CE, et al. Smoking status as a potential confounder in the study of brain structure in schizophrenia. J Psychiatr Res. 2014;50:84-91.

48. Kay SR, Fiszbein A, Opler LA. The positive and negative syndrome scale (PANSS) for schizophrenia. Schizophr Bull. 1987;13(2):261-276.

49. Nuechterlein KH, et al. The MATRICS Consensus Cognitive Battery, part 1: test selection, reliability, and validity. Am J Psychiatry. 2008;165(2):203-213.

50. Kern RS, et al. The MATRICS Consensus Cognitive Battery, part 2: co-norming and standardization. Am J Psychiatry. 2008;165(2):214-220.

51. Green MF, et al. Functional co-primary measures for clinical trials in schizophrenia: results from the MATRICS Psychometric and Standardization Study. Am J Psychiatry. 2008;165(2):221-228.

52. Woods SW. Chlorpromazine equivalent doses for the newer atypical antipsychotics. J Clin Psychiatry. 2003;64(6):663-667.

53. Ibi D, et al. Antipsychotic-induced Hdac2 transcription via NF- $\mathrm{KB}$ leads to synaptic and cognitive side effects. Nat Neurosci. 2017;20(9):1247-1259.

54. de la Fuente Revenga M, et al. Chronic clozapine treatment restrains via $\mathrm{HDAC} 2$ the performance 
of mGlu2 receptor agonism in a rodent model of antipsychotic activity [published online ahead of print July 7, 2018]. Neuropsychopharmacology. https://doi.org/10.1038/s41386-018-0143-4.

55. Kim DI, et al. Dysregulation of working memory and default-mode networks in schizophrenia using independent component analysis, an fBIRN and MCIC study. Hum Brain Mapp. 2009;30(11):3795-3811.

56. Buckner RL. The cerebellum and cognitive function: 25 years of insight from anatomy and neuroimaging. Neuron. 2013;80(3):807-815.

57. Krienen FM, Buckner RL. Segregated fronto-cerebellar circuits revealed by intrinsic functional connectivity. Cereb Cortex. 2009;19(10):2485-2497.

58. Foley AG, Cassidy AW, Regan CM. Pentyl-4-ynVPA, a histone deacetylase inhibitor, ameliorates deficits in social behavior and cognition in a rodent model of autism spectrum disorders. Eur J Pharmacol. 2014;727:80-86.

59. Blokland GAM, et al. The Genetics of Endophenotypes of Neurofunction to Understand Schizophrenia (GENUS) consortium: a collaborative cognitive and neuroimaging genetics project. Schizophr Res. 2018;195:306-317.

60. Bramham CR, Messaoudi E. BDNF function in adult synaptic plasticity: the synaptic consolidation hypothesis. Prog Neurobiol. 2005;76(2):99-125.

61. Duclot F, Kabbaj M. The role of early growth response 1 (EGR1) in brain plasticity and neuropsychiatric disorders. Front Behav Neurosci. 2017;11:35.

62. Yamada K, et al. Genetic analysis of the calcineurin pathway identifies members of the EGR gene family, specifically EGR3, as potential susceptibility candidates in schizophrenia. Proc Natl Acad Sci U S A. 2007;104(8):2815-2820.

63. Kimoto S, Bazmi HH, Lewis DA. Lower expression of glutamic acid decarboxylase 67 in the prefrontal cortex in schizophrenia: contribution of altered regulation by Zif268. Am J Psychiatry. 2014;171(9):969-978.

64. Haggarty SJ, Perlis RH. Translation: screening for novel therapeutics with disease-relevant cell types derived from human stem cell models. Biol Psychiatry. 2014;75(12):952-960.
65. Haggarty SJ, Silva MC, Cross A, Brandon NJ, Perlis RH. Advancing drug discovery for neuropsychiatric disorders using patient-specific stem cell models. Mol Cell Neurosci. 2016;73:104-115.

66. Thompson PM, et al. The ENIGMA Consortium: large-scale collaborative analyses of neuroimaging and genetic data. Brain Imaging Behav. 2014;8(2):153-182.

67. First MB, Spitzer RL, Gibbon M, Williams JBW. Structured Clinical Interview for DSM-IV-TR Axis I Disorders, Research Version, Patient Edition. (SCID-I/P). New York, New York, USA: Biometrics Research, New York State Psychiatric Institute; 2007.

68. First MB et al. Structured Clinical Interview for DSM-IV-TR Axis I Disorders, Research Version, Non-Patient Edition. (SCID-I/NP). New York, New York, USA: Biometrics Research, New York State Psychiatric Institute; 2002.

69. Hollingshead A. Four Factor Index of Social Status. Unpublished manuscript. Yale University, New Haven, Connecticut, USA; 1975.

70. Catana C, et al. Toward implementing an MRIbased PET attenuation-correction method for neurologic studies on the MR-PET brain prototype. J Nucl Med. 2010;51(9):1431-1438.

71. Tisdall MD, Reuter M, Qureshi A, Buckner RL, Fischl B, van der Kouwe AJW. Prospective motion correction with volumetric navigators (vNavs) reduces the bias and variance in brain morphometry induced by subject motion. Neuroimage. 2016;127:11-22.

72. Tisdall MD, Hess AT, Reuter M, Meintjes EM, Fischl B, van der Kouwe AJ. Volumetric navigators for prospective motion correction and selective reacquisition in neuroanatomical MRI. Magn Reson Med. 2012;68(2):389-399.

73. Izquierdo-Garcia D, et al. An SPM8-based approach for attenuation correction combining segmentation and nonrigid template formation: application to simultaneous PET/MR brain imaging. J Nucl Med. 2014;55(11):1825-1830.

74. Chonde DB, Izquierdo-Garcia D, Chen K, Bowen SL, Catana C. Masamune: a tool for automatic dynamic PET data processing, image reconstruction and integrated PET/MRI data analysis.
EJNMMI Phys. 2014;1(Suppl 1):A57.

75. Chonde DB. Improved PET Data Quantification in Simultaneous PET/MR Neuroimaging. Harvard University, Graduate School of Arts Sciences. https://dash.harvard.edu/handle/1/17467219. Accessed November 13, 2018.

76. Fischl B, et al. Whole brain segmentation: automated labeling of neuroanatomical structures in the human brain. Neuron. 2002;33(3):341-355.

77. Woolrich MW, et al. Bayesian analysis of neuroimaging data in FSL. Neuroimage. 2009; 45(1 suppl):S173-S186.

78. Smith SM, et al. Advances in functional and structural MR image analysis and implementation as FSL. Neuroimage. 2004;23 Suppl 1:S208-S219.

79. Jenkinson M, Beckmann CF, Behrens TE, Woolrich MW, Smith SM. FSL. Neuroimage. 2012;62(2):782-790.

80. Jenkinson M, Bannister P, Brady M, Smith S. Improved optimization for the robust and accurate linear registration and motion correction of brain images. Neuroimage. 2002;17(2):825-841.

81. Jenkinson M, Smith S. A global optimisation method for robust affine registration of brain images. Med Image Anal. 2001;5(2):143-156.

82. Andersson JLR, Jenkinson M, Smith S. Non-Linear registration, aka spatial normalisation. FMRIB Technical Report TRO7JA2. Oxford, United Kingdom: Oxford Centre for Functional Magnetic Resonance Imaging of the Brain (FMRIB); 2007.

83. Loggia ML, et al. Evidence for brain glial activation in chronic pain patients. Brain. 2015; 138(pt 3):604-615.

84. Zürcher NR, et al. Increased in vivo glial activation in patients with amyotrophic lateral sclerosis: assessed with [(11)C]-PBR28. Neuroimage Clin. 2015;7:409-414.

85. Yendiki A, et al. Multi-site characterization of an fMRI working memory paradigm: reliability of activation indices. Neuroimage. 2010;53(1):119-131.

86. Wey HY, et al. Simultaneous fMRI-PET of the opioidergic pain system in human brain. Neuroimage. 2014;102(pt 2):275-282.

87. Mai JK, Paxinos G, Voss T. Atlas of the Human Brain. 3rd ed. San Diego, California, USA: Academic Press; 2007. 